\title{
Commentaries
}

\section{Putting pancreatic cell plasticity to the test}

\author{
Jorge Ferrer, Mercè Martín, and Joan Marc Servitja \\ Genomic Programming of Beta Cells Laboratory, Institut d'Investigacions Biomèdiques August Pi i Sunyer, \\ Endocrinology Unit, Hospital Clínic de Barcelona, Barcelona, Spain.
}

\begin{abstract}
Diabetes results from the absolute or relative deficiency of insulin-producing $\beta$ cells. The prospect that non- $\beta$ pancreatic cells could be harnessed to become $\beta$ cells has led to interest in understanding the plasticity of pancreatic cells. Recent studies, however, have shown that adult $\beta$ cells are largely derived from preexisting $\beta$ cells. In this issue of the JCI, Desai et al. show that acinar cells, the major cell type in the pancreas, do not contribute to new $\beta$ cells formed during pancreatic regeneration (see the related article beginning on page 971). These studies suggest that the fate of adult pancreatic cell lineages is immutable. However, also in this issue of the JCI, Collombat et al. demonstrate that inducing a single transcription factor named Arx in adult $\beta$ cells causes these cells to undergo massive transdifferentiation into $\alpha$ and pancreatic polypeptide endocrine cells (see the related article beginning on page 961). This finding points to an unexpected plasticity of postnatal pancreatic endocrine cells.
\end{abstract}

Two experimental strategies have emerged in the past years to restore $\beta$ cell mass in type 1 diabetes. One is to activate a regenerative response in vivo, the other to generate new $\beta$ cells in vitro. Both approaches require an understanding of how new $\beta$ cells are formed.

For many years, the conventional wisdom has been that adult $\beta$ cells have limited, if any, capacity to regenerate. This conception was based in part on the fact that transient treatment with $\beta$ cell toxins like streptozotocin or alloxan leads to the destruction of $\beta$ cells and permanent diabetes mellitus. However, healthy $\beta$ cells clearly grow in response to increased demand, for example, in obesity or pregnancy $(1,2)$. Furthermore, studies have demonstrated that $\beta$ cells undergo robust regeneration after diverse forms of injury, including subtotal pancreatectomy, pancreatic duct ligation, or EGF and gastrin treatment following alloxan-induced diabetes (reviewed in ref. 3). While these models do not necessarily reflect the pathogenesis of human diabetes, they show that $\beta$ cells possess the natural ability to regenerate.

Nonstandard abbreviations used: Arx, aristalessrelated homeobox; LIF, leukemia inhibitory factor; Ngn3, neurogenin 3; Pax4, paired box gene 4; Pdx1, pancreatic and duodenal homeobox $1 ; \mathrm{PP}$, pancreatic polypeptide.

Conflict of interest: The authors have declared that no conflict of interest exists.

Citation for this article: J. Clin. Invest. 117:859-862 (2007). doi:10.1172/JCI31749.
One of the most important challenges in this field is to determine the cellular origin of newly formed $\beta$ cells during regeneration. An attractive idea is that regeneration recapitulates embryonic development. It is now known that pancreatic and duodenal homeobox 1-positive $\left(\mathrm{Pdx} 1^{+}\right)$precursor cells of the early embryonic pancreas give rise to all epithelial lineages of the adult pancreas, suggesting the existence of a common pancreatic precursor cell (4). It is also known that $\beta$ cells arise from ductal structures during embryonic development $(5,6)$. On the other hand, it has been shown that after diverse forms of $\beta$ cell injury in adults, clusters of $\beta$ cells appear in the vicinity of pancreatic ducts, while Pdx1 levels in duct cells often increase $(3,7)$. This was often taken to indicate that $\beta$ cells in such circumstances are derived from pancreatic duct cells, as occurs during embryogenesis. Other candidate origins of regenerating $\beta$ cells have been proposed, most notably acinar cells, as discussed below. However, proving (or disproving) the contribution of any potential source of new $\beta$ cells requires some form of cell lineage tracing.

A Cre/loxP-based lineage tracing study recently provided a breakthrough in the field, showing that throughout the lifetime of an adult mouse, new $\beta$ cells are largely generated from the replication of $\beta$ cells (8). Similarly, following partial pancreatectomy, most $\beta$ cells were also derived from preexisting $\beta$ cells (8). As discussed elsewhere (3), this study could not rule out the possibility that other pancreatic cells give rise to $\beta$ cells after more robust regeneration responses (or even in small amounts in the tested conditions), but it has clearly set the standard for future studies addressing the origin of new $\beta$ cells.

\section{Acinar cells as a source of new $\boldsymbol{\beta}$ cells?}

In this issue of the JCI, Desai et al. (9) tested the contribution of acinar cells to new $\beta$ cells formed during pancreatic regeneration. As the most abundant cell of the pancreas, acinar cells could provide a useful source of insulin-producing endocrine cells. In vitro, adding EGF and leukemia inhibitory factor (LIF) to exocrine cells promotes the formation of new $\beta$ cells at a rate that cannot be accounted for by proliferation of contaminating $\beta$ cells (10). Similarly, EGF plus nicotinamide causes cultured acinar cells, genetically labeled with amylase promoter-driven Cre recombinase, to differentiate into insulin-producing cells (11). Furthermore, there have been indirect indications that acinar to $\beta$ cell differentiation may occur in vivo (12). Desai, Stoffers, and colleagues have now created a transgenic mouse model in which the elastase promoter drives the expression of a hormone-inducible form of Cre selectively in pancreatic acinar cells (9). Upon treatment of adult mice with hormone, Cre activity caused the indelible activation of the $\beta$-galactosidase reporter gene only in acinar cells. The authors then induced $\beta$ cell regeneration by various methods (pancreatectomy and exendin-4 treatment, duct ligation, or pancreatitis). Subsequent examination of $\beta$ cells revealed that none expressed the reporter gene, indicating that acinar cells do not give rise to new $\beta$ cells during regeneration. As the authors carefully point out, this does not disprove that acinar cells can be harnessed to produce insulin using in vitro strategies, as previously reported $(10,11)$. In fact, the apparent conflict between the in vivo and in vitro models is not entirely surprising, because the maintenance of differentiated cellular states is not an entirely cell-autonomous process. Placing cells outside of their natural niche and allowing exposure to differ- 


\section{A Embryonic development}

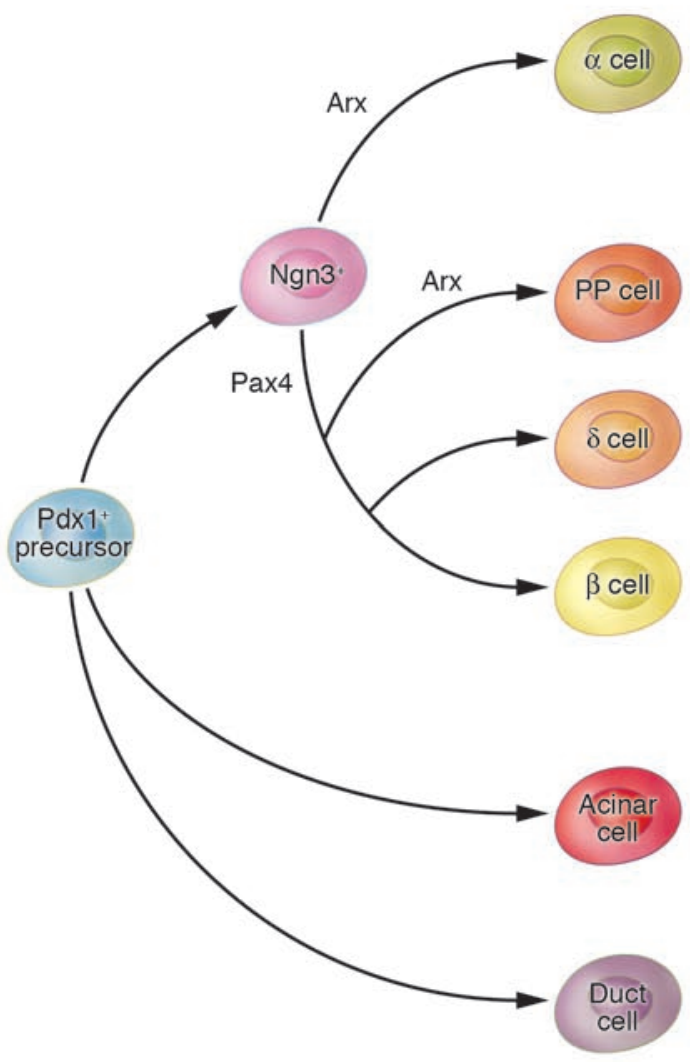

B Postnatal in vivo plasticity

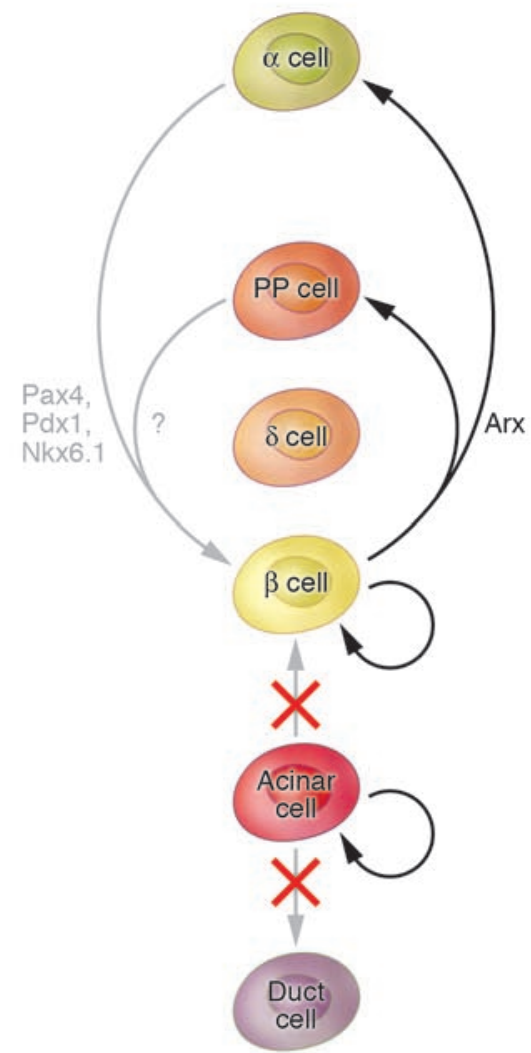

Figure 1

Lineage relationships and plasticity of the embryonic and adult pancreas. (A) All differentiated pancreatic epithelial lineages are derived from $\mathrm{Pdx} 1^{+}$precursor cells of the early embryonic pancreas. A subset of $\mathrm{Pdx} 1^{+}$precursors expressing Ngn3 become committed to the endocrine lineage. The transcription factors Pax 4 and Arx further specify $\beta$ versus $\alpha$ endocrine cells. (B) Given the shared developmental origin, several studies have addressed the possible interconversion of adult pancreatic lineages. For example, in vitro studies indicate that acinar cells can be differentiated into ductal or insulin-producing cells $(10,11)$. In this issue of the $J C l$, Desai et al. (9) have now demonstrated that during regeneration of the pancreas, acinar cells proliferate but do not give rise to $\beta$ cells or duct cells. Together with an earlier study showing that adult $\beta$ cells are largely derived from preexisting $\beta$ cells (8), these findings suggest that in vivo, the plasticity of differentiated pancreatic cells is very limited. However, another study in this issue of the $\mathrm{JCl}$ (13) provides a clear example of pancreatic cell plasticity in vivo, showing that inducible misexpression of the transcription factor Arx in adult mature $\beta$ cells causes transdifferentiation into $\alpha$ and PP endocrine cells. This illustrates how knowledge of developmental transcriptional mechanisms can be exploited to change cellular fates in the adult pancreas. It is conceivable, for example, that misexpression of pro- $\beta$ cell specification factors such as Pax4 might be employed to generate $\beta$ cells from $\alpha$ cells. Nkx6.1, NK6 transcription factor related, locus 1.

ent intercellular signals can have profound consequences on cellular plasticity and differentiation. In summary, the potential for acinar cells to serve as a source of autologous insulin-producing cells remains to be fully examined, although the study by Desai et al. (9) provides a clear message that acinar to $\beta$ cell transdifferentiation is not a prominent mechanism of $\beta$ cell regeneration in vivo.

\section{A case of pancreatic endocrine cell alchemy}

While lineage tracing studies of adult acinar and $\beta$ cells suggest that in vivo postnatal pancreatic cell fates are irreversibly fixed, another study from Collombat and colleagues in this issue of the JCI provides a remarkable example of cellular plasticity in the adult pancreas (13).

One of the most interesting aspects of the study by Collombat et al. (13) is that it exploits the paradigm of the transcriptional regulatory mechanisms of the developing pancreas to manipulate adult cell plasticity $(14,15)$ (Figure 1$)$. It is currently known that the transcription factor neurogenin $3(\mathrm{Ngn} 3)$ orchestrates the regulatory network that gives rise to pancreatic endocrine cells (16). Subsequent regulatory checkpoints refine the specification of endocrine cell subtypes (17-20).
For example, it was previously shown that the ablation of the gene encoding the transcription factor paired box gene 4 (Pax4) causes a complete block of embryonic $\beta$ cell formation and a concomitant increase in the number of glucagon-producing $\alpha$ cells (18). In contrast, deletion of the gene encoding the transcription factor aristaless-related homeobox (Arx) causes the opposite phenotype: no $\alpha$ cells are formed, but $\beta$ cell generation increases (17). This led to the hypothesis that Arx and Pax 4 are mutually exclusive regulators that specify major pancreatic endocrine cell subtypes (Figure 1) (17). However, because these studies were based 
on loss of function, they could not prove that the factors are indeed sufficient to generate such effects. For this reason Collombat et al. (13) chose to force the expression of the pro- $\alpha$ cell factor Arx in endodermal precursors or endocrine progenitors of the embryonic pancreas. This prevented the formation of $\beta$ cells during pancreatic development and increased the number of $\alpha$ cells. Intriguingly, Arx misexpression also caused an increase in the number of pancreatic polypeptide (PP) cells. This was unexpected, because Pax4 $\mathrm{KO}$ embryos, in which Arx expression prevails in endocrine progenitor cells, do not exhibit increased PP cell formation (17). This finding may reflect that Arx specifies PP cell formation only in cells previously exposed to Pax4 (Figure 1). In summary, these experiments clearly showed that Arx is sufficient for suppressing the $\beta$ cell fate. It is also sufficient for specifying $\alpha$ cells, although this effect is not as selective as anticipated from the KO studies.

In the second part of the same study (13), Arx misexpression was induced in $\beta$ cells. In one set of experiments this was done postnatally, long after $\beta$ cells had developed. This resulted in the remarkable finding that within just a few days, normal, healthy $\beta$ cells lost all traces of $\beta$ cell markers and became either PP or $\alpha$ cells. Importantly, the authors employed genetic lineage tracing to demonstrate that there was a true transformation between endocrine cell subtypes, which does not occur under normal conditions (4).

The recapitulation of developmental transcriptional mechanisms to induce transdifferentiation as demonstrated by Collombat et al. (13) is very appealing, but it faces theoretical hurdles. One is that once terminal differentiation occurs, epigenetic mechanisms are thought to restrict fate changes. Another is that developmental gene networks are thought to operate more like complex systems than as simple lineal cascades, making it difficult to obtain clearly directed effects from the manipulation of a single factor. It is thus remarkable that a single transcription factor can entirely reprogram the fate of a healthy differentiated $\beta$ cell (13). Previous studies had shown a similar $\beta$ to $\alpha$ cell transition in INS- $1 \beta$ cell lines after inhibition of $\mathrm{Pdx} 1$, although the mechanisms that control differentiation in tumor cell lines can be profoundly different, as exemplified by the fact that INS- 1 sublines can coexpress both insulin and glucagon genes (21). The deletion of Pdx1 in insulin-producing cells in mice also causes $\beta$ cell loss and replacement by $\alpha$ cells (22), but theoretically this could be because the loss of $\beta$ cells can lead to increased $\alpha$ cell growth. The Collombat et al. study is substantial in that it employed lineage tracing to conclusively demonstrate transdifferentiation between distinct pancreatic cell types in vivo.

This unexpected discovery raises several important questions. First, the results suggest that the epigenome of adult pancreatic endocrine cells is permissive of reprogramming to alternate endocrine subtypes. If this is true, could misexpression of Pax 4 in $\alpha$ cells analogously cause transdifferentiation of $\alpha$ cells into $\beta$ cells? If not, what other DNA-binding proteins need to be induced or suppressed? Because $\alpha$ cells are not destroyed in type 1 diabetes, this would provide a conceptually simple approach to therapeutically increase $\beta$ cell mass. A more challenging possibility is that even if acinar cells do not normally contribute to the $\beta$ cell population after regeneration, in vivo transdifferentiation may be achieved using artificial strategies that mimic embryonic endocrine differentiation. This study also raises interesting mechanistic questions. Although $\beta$ and $\alpha$ cells share several transcription factors, they differ in the content of other regulatory target genes. It is striking that even in a nonphysiological setting, a single transcription factor can simultaneously cause the inactivation of $\beta$ cell-specific genes and activate $\alpha$ cell- and PP cell-specific genes. One possible explanation is that a major function of Arx in the adult transdifferentiation model is to suppress either one or a combination of $\beta$ cell-specific transcription factors. PP and $\alpha$ cells in this context would largely reflect default programs for endocrine cells that do not express other endocrine subtype-specific regulators. Regardless of the exact mechanisms involved, the demonstration that there is no true "point of no return" in adult pancreatic endocrine cells - and the conceptual simplicity with which it was achieved - strongly hints that non- $\beta$ pancreatic endocrine cells are a plausible future source of $\beta$ cells.

\section{Acknowledgments}

The authors' research is supported by the Juvenile Diabetes Research Foundation, the European Union Sixth Framework Programme, Instituto de Salud Carlos III, the European Foundation for the Study of Diabetes, and the Ministerio de Educación y Ciencia.

Address correspondence to: Jorge Ferrer, Endocrinology Unit, Hospital Clinic de Barcelona, Genomic Programming of Beta Cells Laboratory, Institut d'Investigacions Biomèdiques August Pi i Sunyer, Villarroel 170, Barcelona 08036, Spain. Phone: 34-932275400-3028; Fax: 34-934516638; E-mail: jferrer@clinic.ub.es.

1. Lingohr, M.K., Buettner, R., and Rhodes, C.J. 2002. Pancreatic beta-cell growth and survival--a role in obesity-linked type 2 diabetes? Trends Mol. Med. 8:375-384.

2. Bonner-Weir, S. 2000. Life and death of the pancreatic beta cells. Trends Endocrinol. Metab. 11:375-378.

3. Bouwens, L., and Rooman, I. 2005. Regulation of pancreatic beta-cell mass. Physiol. Rev. 85:1255-1270.

4. Herrera, P.L. 2000. Adult insulin- and glucagonproducing cells differentiate from two independent cell lineages. Development. 127:2317-2322.

5. Pictet, R.L., Clark, W.R., Williams, R.H., and Rutter, W.J. 1972. An ultrastructural analysis of the developing embryonic pancreas. Dev. Biol. 29:436-467.

6. Maestro, M.A., et al. 2003. Hnf6 and Tcf2 (MODY5) are linked in a gene network operating in a precursor cell domain of the embryonic pancreas. Hum. Mol. Genet. 12:3307-3314.

7. Bonner-Weir, S., Baxter, L.A., Schuppin, G.T., and Smith, F.E. 1993. A second pathway for regeneration of adult exocrine and endocrine pancreas. A possible recapitulation of embryonic development. Diabetes. 42:1715-1720.

8. Dor, Y., Brown, J., Martinez, O.I., and Melton, D.A. 2004. Adult pancreatic beta-cells are formed by self-duplication rather than stem-cell differentiation. Nature. 429:41-46.

9. Desai, B.M., et al. 2007. Preexisting pancreatic acinar cells contribute to acinar cell, but not islet $\beta$ cell, regeneration. J. Clin. Invest. 117:971-977. doi:10.1172/JCI29988.

10. Baeyens, L., et al. 2005. In vitro generation of insulin-producing beta cells from adult exocrine pancreatic cells. Diabetologia. 48:49-57.

11. Minami, K., et al. 2005. Lineage tracing and characterization of insulin-secreting cells generated from adult pancreatic acinar cells. Proc. Natl. Acad. Sci. U. S. A. 102:15116-15121.

12. Lardon, J., Huyens, N., Rooman, I., and Bouwens, L. 2004. Exocrine cell transdifferentiation in dexamethasone-treated rat pancreas. Virchows Arch. 444:61-65.

13. Collombat, P., et al. 2007. Embryonic endocrine pancreas and mature $\beta$ cells acquire $\alpha$ and PP cell phenotypes upon Arx misexpression. J. Clin. Invest. 117:961-970. doi:10.1172/JCI29115.

14. Murtaugh, L.C. 2007. Pancreas and beta-cell development: from the actual to the possible. Development. 134:427-438.

15. Servitja, J.M., and Ferrer, J. 2004. Transcriptional networks controlling pancreatic development and beta cell function. Diabetologia. 47:597-613.

16. Gradwohl, G., Dierich, A., LeMeur, M., and Guillemot, F. 2000. neurogenin 3 is required for the development of the four endocrine cell lineages of the pancreas. Proc. Natl. Acad. Sci. U. S. A. 97:1607-1611.

17. Collombat, P., et al. 2003. Opposing actions of Arx and Pax4 in endocrine pancreas development. Genes Dev. 17:2591-2603.

18. Sosa-Pineda, B., Chowdhury, K., Torres, M., Oliver, G., and Gruss, P. 1997. The Pax4 gene is essential for differentiation of insulin-producing beta cells 
in the mammalian pancreas. Nature. 386:399-402. 19. Prado, C.L., Pugh-Bernard, A.E., Elghazi, L., SosaPineda, B., and Sussel, L. 2004. Ghrelin cells replace insulin-producing beta cells in two mouse models of pancreas development. Proc. Natl. Acad. Sci.U.S. A. 101:2924-2929.
20. Sander, M., et al. 2000. Homeobox gene Nkx6.1 lies downstream of $\mathrm{Nkx} 2.2$ in the major pathway of beta-cell formation in the pancreas. Development. 127:5533-5540.

21. Wang, H., et al. 2001. Pdx1 level defines pancreatic gene expression pattern and cell lineage differentia- tion. J. Biol. Chem. 276:25279-25286.

22. Ahlgren, U., Jonsson, J., Jonsson, L., Simu, K., and Edlund, H. 1998. beta-cell-specific inactivation of the mouse Ipf $1 / \mathrm{Pdx} 1$ gene results in loss of the beta-cell phenotype and maturity onset diabetes. Genes Dev. 12:1763-1768.

\section{HIF-1 and HIF-2: working alone or together in hypoxia?}

Peter J. Ratcliffe

Nuffield Department of Medicine, University of Oxford, Oxford, United Kingdom.

Erythropoietin (EPO) is the hormonal regulator of red cell production and provided the paradigm for oxygen-regulated gene expression that led to the discovery of hypoxia-inducible factor (HIF). In this issue of the JCI, Rankin and colleagues show, using targeted gene inactivation, that induction of Epo expression in murine liver is dependent on the integrity of HIF-2 $\alpha$, and not HIF-1 $\alpha$ (see the related article beginning on page 1068). These results demonstrate distinct functions for different HIF- $\alpha$ isoforms that could potentially be exploited in therapeutic approaches to anemia.

Erythropoietin (EPO) is a hematopoietic growth factor that, by regulating production of red cells, controls one of the key determinants of physiological oxygen homeostasis, blood oxygen-carrying capacity. In the adult, EPO is produced mainly by the kidneys but also by the liver, and recombinant EPO has been established as the mainstay of treatment of certain types of anemia, particularly anemia associated with chronic kidney disease. In response to severe hypoxia, levels of EPO mRNA and EPO protein production by subsets of cells within the kidneys and liver can increase 1,000-fold or more. This striking response was an initial focus for studies of oxygen-regulated gene expression, and hypoxia-inducible factor (HIF) was defined as the central transcriptional mediator of this process (1). When investigators first probed the molecular pathways underlying oxygen-regulated transcription of the EPO gene, an entirely unanticipated finding was that the pathways involved were widespread and not confined to cells in the kidneys and liver that respond to hypoxia by

Nonstandard abbreviations used: CCRC, clear cell renal carcinoma; EPO, erythropoietin; HIF, hypoxiainducible factor; HRE, hypoxia response element; pVHL, von Hippel-Lindau protein; VHL, von Hippel-Lindau.

Conflict of interest: The author is a founding scientist of ReOx Ltd.

Citation for this article: J. Clin. Invest. 117:862-865 (2007). doi:10.1172/JCI31750. the production of EPO (2). It is now recognized that the HIF system operates in essentially all cells and can directly or indirectly regulate hundreds of genes whose patterns of response to hypoxia and whose physiological functions are quite distinct from those of EPO. These include genes involved in functionally diverse responses such as cell motility and differentiation, matrix metabolism, and angiogenesis; genes with contrasting kinetic responses to hypoxia such as the massively inducible EPO gene versus the modestly inducible genes encoding glycolytic enzymes; and genes encoding proteins with apparently opposing physiological functions, such as growth factors and proapoptotic mediators (3). The unexpected pleiotropism of the HIF transcriptional cascade presumably underpins the complexity of oxygen homeostasis, but it begs a fundamental question as to how the system could orchestrate such diverse responses.

\section{The oxygen-sensitive HIF hydroxylase pathway}

HIF is an $\alpha / \beta$ heterodimer that binds hypoxia response elements (HREs) at target gene loci under hypoxic conditions (Figure $1)$. In the presence of oxygen, HIF is inactivated by posttranslational hydroxylation of specific amino acid residues within its $\alpha$ subunits. Prolyl hydroxylation promotes interaction with the von Hippel-Lindau protein (pVHL) E3 ubiquitin ligase complex and proteolytic inactivation by proteasomal degradation, while asparaginyl hydroxylation blocks coactivator recruitment. These hydroxylation steps are catalyzed by a set of non-heme Fe(II)- and 2-oxoglutaratedependent dioxygenases whose absolute requirement for molecular oxygen confers sensitivity to hypoxia (4). So, how might such an apparently simple pathway transduce hypoxia signaling with the precision required to maintain physiological oxygen homeostasis, and how might the existence of different HIF- $\alpha$ isoforms contribute to this process? Answers to these questions should interest a range of medical scientists; both those seeking to understand the basic biology of oxygen homeostasis and those seeking to assess the feasibility of manipulating hypoxia signaling pathways for therapeutic purposes.

\section{HIF-1 $\alpha$ and HIF-2 $\alpha$ isoforms}

HIF-1 $\alpha$ was the original HIF isoform identified by affinity purification using oligonucleotides from the EPO locus (1), while HIF-2 $\alpha$ and HIF-3 $\alpha$ were identified by homology searches or screens for interaction partners with HIF- $1 \beta$. HIF- $3 \alpha$ is the more distantly related isoform and, in certain splicing arrangements, encodes a polypeptide that antagonizes HRE-dependent gene expression. However, HIF- $1 \alpha$ and HIF- $2 \alpha$ are closely related, and both activate HRE-dependent gene transcription (3). Nevertheless, knockout studies in mice demonstrate that HIF-1 $\alpha$ and HIF- $2 \alpha$ play nonredundant roles, and inactivation of each one results in a distinctly different phenotype. This may result, in part, from differences in tissue-specific and temporal patterns of induction of each isoform (5-7), but, not uncommonly, both isoforms are expressed within a given cell type, and the results of several studies, including those 